\title{
Entrincheiramento Organizacional: Construção e Validação da Escala
}

\author{
Organizational Entrenchment: Scale Development and Validation
}

\author{
Ana Carolina de Aguiar Rodrigues ${ }^{*}, a$ \& Antonio Virgilio Bittencourt Bastos ${ }^{b}$ \\ ${ }^{a}$ Universidade Nove de Julho, São Paulo, Brasil \& ${ }^{b}$ Universidade Federal da Bahia, Salvador, Brasil
}

\begin{abstract}
Resumo
O presente trabalho teve como objetivo construir e validar a escala de entrincheiramento organizacional, construto de mesma base teórica do comprometimento de continuação. Três dimensões foram propostas: ajustamentos à posição social (APS), arranjos burocráticos impessoais (ABI) e limitação de alternativas (LA). Os dados coletados com 721 trabalhadores foram submetidos a análises exploratórias e confirmatórias para a avaliação psicométrica da escala. Os resultados indicam estabilidade, generalizabilidade e alta consistência interna dos três fatores, formados pelos vinte e dois itens restantes, e respaldam a decisão pela estrutura tridimensional. Estudos futuros com a escala validada poderão contribuir para um maior refinamento conceitual e empírico do entrincheiramento com a organização.

Palavras-chave: Entrincheiramento organizacional, vínculos com a organização, construção e avaliação psicométrica de escala, análise fatorial confirmatória.
\end{abstract}

\begin{abstract}
The objective of this work was to develop and validate an organizational entrenchment scale which is a construct on the same theoretical base of continuance commitment. Three dimensions were defined: individual adjustment to social positions (ASP), impersonal bureaucratic arrangements (IBA) and limitation of alternatives (LA). Data collected from 721 workers were subjected to exploratory and confirmatory analyses in order to conduct a psychometric evaluation of the scale. Results indicate stability, potential for generalization and strong internal consistency across the three dimensions formed by the remaining twenty-two items, and they also support the choice of a three-component structure. Future studies using this validated scale will contribute for further conceptual and empirical refinement of the organizational entrenchment concept.

Keywords: Organizational entrenchment, organizational attachments, scale development and psychometric evaluation, confirmatory factor analysis.
\end{abstract}

$\mathrm{Na}$ área da Psicologia Organizacional e do Trabalho, os estudos sobre comportamento micro-organizacional foram impulsionados pela compreensão de que o comportamento humano tem um papel fundamental no desenvolvimento e no funcionamento das organizações (Gondim, Borges-Andrade, \& Bastos, 2010). Nesse campo, as pesquisas sobre vínculos no trabalho foram estimuladas pelo entendimento de que as organizações dependem essencialmente da presença, articulação e vinculação de pessoas aos seus objetivos, trabalhos e equipes, que por sua vez impactam no bem-estar dos indivíduos. Em meio século de existência, essa tradição de pesquisa acumulou avanços significativos de modelos

\footnotetext{
"E-mail: anacarolina.ar@gmail.com e virgilio@ufba.br. $\mathrm{O}$ artigo é proveniente da dissertação de mestrado da primeira autora. Agradecemos ao Conselho Nacional de Desenvolvimento Científico e Tecnológico (CNPq) e à Fundação de Amparo à Pesquisa do Estado da Bahia (Fapesb) pelo apoio financeiro para a realização da pesquisa.
}

teóricos e evidências empíricas, mas não o fez sem o acúmulo igualmente significativo de problemas conceituais e metodológicos.

$\mathrm{O}$ conceito de comprometimento, que tem recebido destaque nas pesquisas sobre vínculos no trabalho, apresenta atualmente um quadro de imprecisão, ocasionado pela ampliação excessiva do seu alcance conceitual. Esse esticamento fez com que passasse a abarcar diferentes vínculos, sendo muitas vezes confundido com seus antecedentes e consequentes (Klein, Molloy, \& Cooper, 2009; Osigweh, 1989). Em especial, as principais discussões sobre problemas empíricos e teóricos referem-se àquele que tem sido mais utilizado nos estudos desse campo: o modelo tridimensional do comprometimento (Meyer, Allen, \& Smith, 1993), formado pelas bases afetiva (identificação e afeto para com a organização), normativa (resultado de normas e valores internalizados) e de continuação (racionalidade instrumental do trabalhador voltada para o cálculo do que seria perdido se deixasse a organização). 
A ampliação conceitual implicada neste modelo tridimensional por um lado afeta a validade e precisão dos estudos sobre comprometimento e, por outro, mascara vínculos que deveriam estar sendo investigados separadamente. $\mathrm{O}$ conceito de entrincheiramento, proposto por K. D. Carson, P. P. Carson e Bedeian (1995) com foco na carreira, abriu a possibilidade de análise de um diferente tipo de vínculo, capaz de explicar um comportamento do trabalhador até então atribuído ao comprometimento: a permanência na organização por necessidade. Segundo os autores, o entrincheiramento na carreira seria a tendência do indivíduo a permanecer em sua ocupação devido aos investimentos que seriam perdidos caso a deixasse e aos custos emocionais que resultariam dessa decisão, potencializados pela percepção de falta de alternativas no mercado de trabalho.

Por ter sido proposto em um momento de efervescência dos debates sobre problemas conceituais e empíricos do vínculo de comprometimento (Rodrigues \& Bastos, 2010), as principais atenções voltadas para o conceito de entrincheiramento na carreira tiveram como objetivo confrontálo com a base de continuação do comprometimento (Blau \& Holladay, 2006; Scheible, Bastos, \& Rodrigues, 2007). Quatro argumentos principais motivaram esses estudos:

1. Ambos os construtos remetem à teoria dos side bets de Becker (1960), segundo a qual o sujeito permanece em uma linha de ação a fim de manter os ganhos já alcançados e reduzir as possibilidades de perda.

2. Possuem subdimensões semelhantes, uma vez que o comprometimento de continuação seria formado pelos fatores sacrifícios pessoais e limitação de alternativas (McGee \& Ford, 1987), e o entrincheiramento, por custos acumulados e limitação de alternativas (Blau, 2001).

3. Os dois vínculos representam uma atitude frente ao comportamento de permanecer na organização, configurando uma relação material e uma permanência por necessidade (Solinger, Olffen, \& Roe, 2008).

4. Ambos apresentam correlações positivas entre si (K. D. Carson et al., 1995; Scheible \& Bastos, 2006) e negativas com comportamentos desejáveis (Cooper-Hakim \& Viswesvaran, 2005; Magalhães, 2008). Tais aspectos permitem questionar se esses construtos não estariam se referindo a um mesmo fenômeno psicossocial.

A necessidade de levar essa análise ao foco das organizações, que tem sido o principal alvo dos debates conceituais sobre comprometimento, motivou a elaboração do quadro teórico do entrincheiramento organizacional. Três bases conceituais foram consideradas: o construto de entrincheiramento na carreira (K. D. Carson et al., 1995), a teoria dos side bets de Becker (1960) e a proposta de Mowday, Porter e Steers (1982) para a terceira fase de desenvolvimento do comprometimento, denominada entrincheiramento. Essas três bases teóricas apresentam uma lógica comum para o vínculo de entrincheiramento: a importância dos investimentos e papéis sociais assumi- dos pelo trabalhador ao longo do tempo, e as recompensas advindas desses comportamentos, que seriam perdidas caso deixasse a organização e dificilmente recuperadas em outro contexto.

Essa lógica originou três dimensões para o entrincheiramento organizacional, sendo as duas primeiras derivadas da teoria de Becker (1960):

- Ajustamentos à posição social (APS): investimentos do indivíduo nas condições necessárias à sua adaptação, a exemplo de treinamentos para desempenho de atividades específicas, tempo para conhecer os processos organizacionais e as atribuições da função, relacionamentos construídos com os colegas, entre outros aspectos que contribuiriam para o seu ajuste e reconhecimento na organização, e que seriam perdidos caso a deixasse;

- Arranjos burocráticos impessoais (ABI): estabilidade financeira e benefícios que seriam perdidos se o indivíduo saísse da organização, como férias e feriados pagos, participação nos lucros, assistência médica, previdência privada, aposentadoria, entre outros;

- Limitação de alternativas (LA): percepção de restrições no mercado de trabalho e de falta de alternativas caso saísse da organização, seja por visualizar déficits em seu perfil profissional, por considerar que a idade desfavorece a reinserção ou outros fatores que possam reduzir a empregabilidade percebida.

Esse último fator, quando relativo à estrutura do comprometimento de continuação, do qual também é constituinte, tem sido alvo de argumentos de que deveria ser tratado como antecedente, e não como parte do comprometimento (Ko, Price, \& Mueller, 1997). Tal proposta baseia-se na noção de que a limitação de alternativas é um determinante direto da permanência por necessidade, que por sua vez não deveria ser considerada um consequente direto do comprometimento, a ponto de ter um critério inserido em seu conceito e em sua medida. No caso do entrincheiramento, ao contrário, entende-se que a percepção de pouca ou nenhuma alternativa seja um fator decisivo para que o indivíduo sinta-se preso à organização. Essa noção não está presente na teoria dos side bets de Becker (1960), mas atravessa todas as dimensões do entrincheiramento organizacional, uma vez que as dimensões APS e ABI podem levar a uma percepção de limitação intrínseca: à medida que o indivíduo se adapta às condições e aos retornos materiais da organização, percebe uma maior restrição do seu perfil e menores oportunidades de emprego em que as condições alcançadas na organização atual estejam também disponíveis. A dimensão LA traz, adicionalmente, uma percepção de limitação extrínseca, condicionada à avaliação que o indivíduo faz das opções disponíveis no mercado.

Rodrigues e Bastos (2011) incluem essa discussão quando propõem o modelo teórico do entrincheiramento organizacional. O modelo do vínculo parte da história do trabalhador na organização, de sua análise dos investimentos 
feitos e do que poderia perder caso a deixasse. Se não percebe grandes custos associados a sua saída, então não está entrincheirado. $\mathrm{O}$ indivíduo pode, ainda, acreditar que há custos, mas perceber outras oportunidades de trabalho que sejam compensatórias. Contudo, se entende que perderá os esforços de ajustamento e os retornos materiais obtidos, e não percebe alternativas de emprego, provavelmente estará entrincheirado. O modelo teórico supõe, também, que o trabalhador entrincheirado pode ou não ter um vínculo afetivo com a organização, variável que impactará em seu comportamento.

O conceito de entrincheiramento organizacional, até então ausente na literatura nacional e internacional (Rodrigues, 2009; Rodrigues \& Bastos, 2011), foi concebido diante da necessidade de ampliar a precisão dos vínculos com a organização e de investigar conceitos que até então eram considerados parte do comprometimento. Diante também da necessidade de elaborar e validar medidas precisas desses vínculos, e de testar o modelo teórico do entrincheiramento, o presente trabalho teve como objetivo construir e validar a escala de entrincheiramento organizacional.

\section{Método}

\section{Construção da Escala}

Elaboração dos Itens. Uma vez que, até então, não havia trabalhos teóricos ou empíricos sobre o entrincheiramento com foco na organização, seu desenvolvimento teórico foi o principal demarcador para a proposição dos itens. Foi realizada uma ampla revisão de escalas já validadas e relacionadas aos fatores sugeridos para o construto, especialmente aquelas construídas para mensurar os side-bets e o comprometimento de continuação, a partir das quais foram selecionados: seis itens provenientes da escala de Siqueira (1995, citado por Bastos, Siqueira, Medeiros, \& Menezes, 2008), dois itens de Meyer et al. (1993), dois itens de Rego (2003), um item de Powell e Meyer (2004) e um item adaptado de K. D. Carson e P. P. Carson (2002). Além desses, dezenove itens foram redigidos pelo grupo de pesquisa (GP), após discussões realizadas com base na estrutura teórica do entrincheiramento organizacional, e nas definições constitutivas e operacionais dos fatores. Finalmente, a medida totalizou 31 itens, dispostos em escala likert de seis pontos (discordo totalmente a concordo totalmente).

Análise dos Juizes. Nove juízes, pesquisadores da área da Psicologia Organizacional e do Trabalho, realizaram a análise semântica dos itens. Os critérios de exclusão dos itens seguiram as orientações de Pasquali (1999), que estabelece como ponto de corte $80 \%$ de concordância entre os juízes. Contudo, o autor ressalva que, no caso de testes com fatores supostamente correlacionados, como é o caso da medida de entrincheiramento organizacional, é provável que os juízes discordem quanto à adequação de determinado item a diferentes fatores, o que deve ser considerado como concordância. Por essa razão, foram mantidos os itens com um percentual de concordância mínimo de $60 \%$ em um dos fatores e ao menos $20 \%$ de concordância em um segundo fator. Dos trinta e um itens indicados inicialmente, vinte e seis foram satisfatórios.

\section{Validação da Escala}

Procedimento de Coleta dos Dados. O instrumento de pesquisa foi aplicado a partir de três diferentes procedimentos de coleta: auto-aplicação do questionário em versão digital (via internet), auto-aplicação em versão impressa ou ainda aplicação sob a forma de entrevista (para trabalhadores de menor escolaridade). Nesse caso, foram utilizados recursos que facilitaram a compreensão das questões, a exemplo de escalas coloridas para discriminação dos níveis de concordância dos respondentes. Os entrevistadores foram previamente treinados para garantir uniformidade de aplicação. Os diferentes meios de acesso aos respondentes tiveram como objetivo alcançar uma amostra diversificada, necessária para os procedimentos de análise e validação da medida de entrincheiramento. Uma vez que em estudos organizacionais nem sempre é viável obter amostras aleatórias, já que as organizações contatadas incubem-se de convidar os trabalhadores, a forma de acesso aos participantes foi por conveniência, a partir de convites a organizações e trabalhadores, quer nos seus ambientes de trabalho, quer acessados em cursos de nível superior, pós-graduação e supletivos, entre outros. A aplicação pela internet viabilizou a distribuição da versão digital do questionário a diferentes trabalhadores, que eram convidados a distribuí-la a outros trabalhadores.

Participantes. Setecentos e vinte e um trabalhadores responderam à pesquisa, dos quais $82,3 \%$ vivem no Nordeste do Brasil e $66 \%$ trabalham em empresas privadas, atuando principalmente em prestação de serviços (30,3\%), comércio $(22,8 \%)$ ou indústrias $(13,2 \%)$. Pouco mais da metade dos participantes são do sexo feminino $(55,3 \%)$, solteiros $(49,4 \%)$ e com menos de trinta e cinco anos $(59,2 \%)$. Com relação à escolaridade, a maioria teve acesso ao curso de nível superior (71,9\%), e uma pequena parcela possui até o segundo grau incompleto $(7,3 \%)$. No que se refere à renda mensal, $19,9 \%$ recebem menos de um salário mínimo, e grande parte da amostra (48,9\%) recebe pagamentos na faixa de um a cinco salários mínimos, cálculo feito com base no valor corrente no período da coleta de dados $(\mathrm{R} \$ 380,00)$. Quanto ao tempo de serviço, $31,2 \%$ da amostra trabalha na organização há menos de dois anos, e $25,7 \%$ tem entre dois e cinco anos de trabalho na organização. Nota-se, portanto, que a amostra tende a uma composição de trabalhadores relativamente jovens, de alta escolaridade, empregados em empresas privadas, com pouco tempo de serviço e com salários mais modestos.

Procedimentos de Análise dos Dados. Para a condução da análise fatorial, foi utilizado o programa estatístico SPSS 13.0. Optou-se por empregar o método de extração PAF (principal axis factoring) e rotação oblíqua. A esco- 
lha do método de extração PAF foi devido ao objetivo de identificar a estrutura latente dos dados com base na variância compartilhada, sem conhecimento prévio das variâncias específica e de erro. Optou-se por uma rotação oblíqua a partir da hipótese de que os fatores do construto investigado apresentam correlações entre si (Hair, Anderson, Tatham, \& Black, 2005). O método rotacional utilizado foi o Promax, com potência (kappa) de valor 4. Para medir a adequação da amostra, foi utilizado o teste KMO (Kaiser-Meyer-Olkin). Para avaliar a adequação dos dados foi utilizado também o teste de esfericidade de Bartlett, que indica a presença de correlações significativas entre as variáveis.

Após a extração dos fatores, foram realizados testes de consistência interna para cada dimensão. Embora o alfa de Cronbach seja frequentemente utilizado como indicador de confiabilidade, seus resultados são afetados pelo número de itens da escala, ou por uma possível redundância entre itens (Clark \& Watson, 1995). Por isso, foram adotados também outros critérios de avaliação da consistência interna, como a correlação item-total e a média de correlação inter-item, satisfatórios quando atingem valores, respectivamente, acima de 0,20 (Menezes, 2006) e no intervalo de 0,15 a 0,50 , devendo aproximarse de 0,50 a média de correlações inter-item de construtos mais específicos e menos complexos (Clark \& Watson, 1995). A validação cruzada foi aplicada em seguida, para avaliar a estabilidade da solução fatorial obtida. Para tanto, a amostra total de participantes do estudo foi dividida em dois grupos, cujas características foram avaliadas, a fim de verificar a existência de possíveis diferenças que poderiam afetar a estrutura fatorial. Foi realizado também um teste da generalizabilidade da estrutura em amostras com características demográficas distintas, conforme sugerido por Laros (2005).

As análises que utilizaram modelagem de equações estruturais foram realizadas com o pacote estatístico AMOS 16.0. O método de estimação empregado foi o da Máxima Verossimilhança (ML), tendo em vista o tamanho da amostra e o caráter moderadamente anormal dos dados. Para os testes dos modelos de mensuração, optouse por adotar novamente a técnica de validação cruzada, seguindo as recomendações de verificar a pertinência das modificações implantadas após a primeira avaliação dos modelos (Hair et al., 2005; Ullman, 2007; Weston \& Gore, 2006). Em seguida às análises dos modelos de mensuração, foram testados os modelos estruturais com a amostra total da pesquisa. Para a avaliação do ajuste dos modelos, foram considerados os seguintes índices e critérios: índices de ajuste comparativo (NFI, IFI, CFI, que devem ser superiores a 0,90 e RMSEA, que deve ser inferior a 0,08$)$; índices de proporção da variância explicada (GFI e AGFI, esperados acima de 0,90 ); índices de parcimônia (PGFI, que deve estar próximo a 1,0, AIC e CAIC, que devem apresentar menores valores em relação ao modelo concorrente) e índice de ajuste basea- do em resíduos (RMR, que deve apresentar valores pequenos).

\section{Resultados}

A seguir, são apresentados inicialmente os resultados das análises exploratórias dos itens, que investigaram a dimensionalidade do construto, a representatividade dos itens na mensuração de cada fator, a consistência interna dos itens por dimensão, a estabilidade e a "generalizabilidade" da estrutura fatorial. Após isso, são apresentados os resultados referentes à etapa confirmatória de análise, em que foram testados modelos alternativos para a verificação e discussão das dimensões do entrincheiramento organizacional.

\section{Análise Fatorial Exploratória}

Antes de iniciar a etapa exploratória, foi inspecionada a matriz de correlação de Pearson para os trinta e um itens inicialmente propostos. Quatro itens, que também haviam sido avaliados negativamente pelos juízes, apresentaram correlações fracas ou não significativas com a maioria das variáveis restantes. Ao retirá-los do modelo fatorial, a média de correlações inter-item resultou em um valor de 0,25 , que indica correlações moderadas entre as variáveis, seguindo as recomendações de Clark e Watson (1995). A média aritmética das correlações item-total foi de 0,47 , com desvio padrão de 0,05 , e o alfa de Cronbach de todo o conjunto foi de 0,90 , revelando fatorabilidade da matriz.

A análise exploratória foi realizada, então, com os 27 itens restantes. O KMO resultou em um valor de 0,916 , que indica alta adequação da amostra. $\mathrm{O}$ teste de esfericidade de Bartlett foi significativo $\left[\chi^{2}(351)=5111,065\right.$, $\rho<0,01]$. Para a extração de fatores, foi considerada a relevância teórica das dimensões e observados os critérios de eigenvalue e teste scree que, embora tenham a tendência a superestimar o número de fatores (Zwick \& Velicer, 1986), são os únicos disponíveis no pacote do SPSS.

Cinco fatores tiveram eigenvalues acima de 1, podendo alcançar uma variância acumulada de aproximadamente $49 \%$. O teste scree revelou que os três primeiros fatores respondem por uma parte maior da variância, e os demais reduzem gradativamente a variância explicada, aproximando-se de uma reta horizontal. Foram inicialmente analisadas as soluções com cinco e quatro componentes, em que fatores adicionais reuniram itens representativos ou apresentaram cargas residuais dos primeiros fatores. Portanto, para evitar a extração de dimensões vazias conceitualmente e buscar uma maior validade do construto, optou-se por extrair três fatores, conforme previsto pela proposição teórica do entrincheiramento organizacional.

Ao considerar o tamanho da amostra e a proporção de casos por item, uma carga fatorial de 0,30 já é considerada significativa (Hair et al., 2005). Foram mantidas, portanto, as variáveis que apresentaram carga fatorial 
acima de 0,30 e que tiveram uma diferença de ao menos 0,10 para cargas dirigidas a outros fatores. Tais critérios levaram à exclusão de mais cinco itens, além dos quatro inicialmente eliminados.

A etapa subsequente consistiu em realizar uma nova análise com os 22 itens restantes, em busca de uma maior precisão da solução fatorial. $\mathrm{O}$ determinante da matriz foi maior do que zero $(0,002)$, a média de correlações inter-item foi de 0,25 e o KMO resultou em um valor de 0,896 . O teste de esfericidade de Bartlett foi significativo $\left[\chi^{2}(231)=4135,385, \rho<0,01\right]$. A análise da consistência interna do conjunto dos itens revelou um alfa de Cronbach de 0,88 e uma média aritmética de correlações item-total de 0,46 , com desvio padrão de 0,06 . A comparação dessa solução com o conjunto dos 27 itens iniciais indica que as eliminações executadas não alteraram a fatorabilidade ou a consistência interna da matriz e ratifica que a retirada dos itens reduziu a redundância da escala, já que estão representados por outras variáveis.

Os três fatores extraídos explicam $43,7 \%$ da variância dos itens e apresentam eigenvalues de 6,22, 1,85 e 1,54, respectivamente. Na Tabela 1, são apresentadas as cargas fatoriais dos itens, índices de consistência interna e variância explicada por cada fator.

Tabela 1

Itens de Entrincheiramento Organizacional e suas Cargas Fatoriais

\begin{tabular}{llll}
\hline Indicadores & APS & LA & ABI \\
\hline
\end{tabular}

ES12 Se eu fosse trabalhar em outra empresa, eu estaria jogando fora todo o esforço que fiz para aprender as tarefas do meu cargo atual.

ES5 Se deixasse essa organização, sentiria como se estivesse desperdiçando anos de dedicação.

ES7 Sair dessa organização significaria, para mim, perder parte importante da minha rede de relacionamentos.

ES9 Se eu fosse trabalhar em outra empresa, eu jogaria fora todos os esforços que fiz para chegar aonde cheguei dentro dessa empresa.

ES8 Um dos motivos pelos quais não mudo de organização são os relacionamentos que já estabeleci com os colegas.

ES10 Se eu fosse trabalhar em outra empresa, eu demoraria a conseguir ser

tão respeitado como sou hoje dentro dessa empresa.

0,61

0,56

0,52

0,51

ES1 Eu investi muito tempo no meu aprendizado dentro dessa organização para

deixá-la agora.

ES11 Se eu fosse trabalhar em outra empresa, eu levaria muito tempo para me acostumar a uma nova função.

IL2 Eu acho que teria poucas alternativas de emprego se deixasse essa organização.

IL4 Mantenho-me nessa organização porque sinto que não conseguiria facilmente entrar em outra organização.

IL5 Mantenho-me nessa organização porque sinto que tenho poucas oportunidades em outras organizações.

ES4 Seria difícil encontrar outro emprego onde eu pudesse usar os conhecimentos de trabalho que adquiri nesta empresa.

IL3 Uma das poucas consequências negativas de deixar essa organização seria a falta de alternativas.

EL4 Meu perfil profissional não favorece minha inserção em outras organizações.

EL3 A especificidade do meu conhecimento dificulta minha inserção em outras organizações.

EB4 Sinto que mudar de empresa colocaria em risco a minha estabilidade financeira.

EB3 Sair dessa organização agora resultaria em perdas financeiras.

EB1 Se eu deixasse essa organização, minha estabilidade seria ameaçada.

EB2 Os benefícios que recebo nessa organização seriam perdidos se eu saísse agora.

EB5 O que me prende a essa organização são os benefícios financeiros que ela me proporciona.

EL6 Não seria fácil encontrar outra organização que me oferecesse o mesmo retorno

financeiro que essa.

EB7 Se eu fosse trabalhar em outra empresa, eu deixaria de receber vários benefícios que essa

empresa oferece aos seus empregados (vale transporte, convênios médicos, vale refeição, etc).

Alfa de Cronbach

Média de correlações inter-item

Variância explicada
0,50

0,49

0,44

0,66

0,63

0,62

0,57

0,56

0,54

0,47

0,76

0,72

0,47

0,47

0,45

0,40

0,36

$\begin{array}{lll}0,80 & 0,79 & 0,77\end{array}$

$0,33 \quad 0,36 \quad 0,32$

$28 \% \quad 8 \% \quad 7 \%$

Notas. Método de extração: Análise de Fatores Comuns. Método de rotação: Promax com normalização Kaizer. Rotação convergida em 6 iterações. Fonte: Dados da pesquisa. 
O primeiro fator, APS, reuniu oito itens referentes aos esforços e investimentos do indivíduo no processo de adaptação à organização, como tempo, conhecimentos adquiridos, status alcançado e redes de relacionamentos formadas. $\mathrm{O}$ fator 2, LA, reuniu sete itens que avaliam a percepção do respondente em relação às oportunidades de emprego fora da organização em que trabalha. Nesse fator, a análise fatorial carregou o item ES4, elaborado inicialmente para APS. O item enfatiza a dificuldade percebida em encontrar outro emprego onde o indivíduo pudesse utilizar os conhecimentos adquiridos. Assim, a ênfase não é no investimento que seria perdido, mas na alternativa limitada de utilizar os conhecimentos adquiridos fora da organização. É provável que esse raciocínio tenha guiado os juízes que atribuíram este item também ao fator LA. O fator 3, ABI, reuniu sete itens referentes aos custos associados à saída da organização, a exemplo de perda de estabilidade financeira e perda de benefícios. Também nesta dimensão foi carregado um item inicialmente elaborado para outro fator, o EL6. Uma avaliação semântica desse item indica que, embora tenha sido construído para a dimensão LA, é pertinente sua continuidade na dimensão $\mathrm{ABI}$, uma vez que é enfatizado o retorno financeiro que não seria recuperado caso o indivíduo deixasse a organização. Além disso, $66,7 \%$ dos juízes haviam apontado esse item como sendo parte de ABI.

Os índices de confiabilidade obtidos foram satisfatórios para os três fatores, indicando que a solução tridimensional é consistente com o quadro teórico do entrincheiramento organizacional. Assim, o indivíduo se ajusta à posição social que ocupa na organização por meio de um processo adaptativo que consiste no estabelecimento do papel a ser desempenhado e formação de relacionamentos profissionais, além da exigência de tempo e recursos para o aprendizado. À medida que esse processo acontece parece mais difícil deixar a organização, pois seria necessário renunciar ao espaço conquistado. Em caso de uma nova inserção, o indivíduo precisaria percorrer novamente todo o caminho de investimentos e ajustes, ainda assim sem garantias de alcançar a mesma posição anterior. Similar a isso, o indivíduo pode analisar o retorno material fornecido pela organização em função do tempo de trabalho dedicado, especialmente os benefícios financeiros, avaliando, talvez, que não conseguiria obter os mesmos benefícios em outras organizações. Tal avaliação pode ser reforçada pela percepção de que não há boas alternativas de trabalho disponíveis no mercado, onde pudesse aproveitar o perfil profissional desenvolvido. Diante desses aspectos, o trabalhador pode entender como sendo mais vantajoso manter o curso de ação adotado até o momento (side bets), a fim de garantir a continuidade de suas conquistas na organização.

Verificou-se, ainda, que há correlações positivas e significativas entre os fatores APS e LA $(r=0,621)$, APS e $\mathrm{ABI}(r=0,512)$ e LA e ABI $(r=0,439)$. Esse resultado fortalece os indícios de coesão do construto e confirmam a hipótese de que os fatores são correlacionados.
Após a realização da análise fatorial exploratória, foram avaliadas a estabilidade e a possibilidade de generalização da estrutura encontrada. Para a realização do primeiro teste, o banco de dados foi dividido aleatoriamente em duas partes, obtendo-se duas sub-amostras independentes de 360 e 361 participantes. Testada a hipótese de igualdade das amostras na previsão do construto, por meio do teste $t$, verificou-se que não há diferenças significativas entre os grupos para as três dimensões avaliadas, condição necessária para a validação cruzada.

Foram realizadas análises exploratórias independentes com os 22 itens mantidos após a análise com a amostra geral e aplicados os mesmos procedimentos e decisões anteriores. Para as duas amostras, o KMO foi maior que 0,86, e o teste de Bartlett foi significativo. A estrutura trifatorial foi obtida em ambos os casos, com variâncias totais semelhantes ( $43 \%$ e $45 \%)$. Os itens carregaram nos mesmos fatores observados na amostra geral, com alfas e médias de correlação inter-item e item-total semelhantes nos dois casos, sendo superiores a $0,76,0,31$ e 0,47 , respectivamente.

Em seguida à validação cruzada, foi investigada a generalizabilidade do modelo por meio da execução de análises exploratórias com duas amostras diferentes em relação ao tempo de serviço dos participantes na empresa atual. A opção por comparar grupos com tempos de empresa distintos foi respaldada pela suposição teórica de que as dimensões do entrincheiramento poderiam variar a depender do tempo investido pelo indivíduo na organização (Becker, 1960; Mowday et al., 1982).

Para realizar a divisão, foi inicialmente calculada a mediana da amostra geral quanto a tempo de empresa e, em seguida, foram separados os participantes em dois grupos, sendo o primeiro composto por 361 profissionais com até 47 meses na empresa, e o segundo, por 356 profissionais com mais de 47 meses. Mais uma vez, foi testada a igualdade entre as respostas, e o teste $t$ revelou diferenças significativas entre os grupos para as dimensões APS $[t(711,3)=3,242, p<0,01]$ e $A B I[t(714,5)=3,152$, $p<0,01]$, mas não para a dimensão $\operatorname{LA}[t(713,8)=1,32$, $p=0,187]$. Os participantes com mais de 47 meses apresentaram médias maiores para APS $[t(711,3)=3,1$, $D P=0,93]$ e para $\mathrm{ABI}[t(714,5)=3,6, D P=1,03]$ do que os participantes com até 47 meses de empresa, que apresentaram médias de $2,8(D P=1,01)$ e $3,3(D P=1,01)$, respectivamente. Tais dados trazem evidências empíricas de que o tempo de empresa impacta no nível de entrincheiramento organizacional. Ao contrário da validação cruzada, cujo requisito é a homogeneidade dos grupos, a avaliação da generalizabilidade é justificada quando permite verificar se a estrutura fatorial é reproduzida em diferentes segmentos da população (Laros, 2005).

Foram aplicados os mesmos procedimentos de análise para os dois grupos. O teste de Bartlett foi significativo, e os valores do KMO estiveram acima de 0,866 para os dois grupos. Consistente com os resultados da validação cruzada, foi obtida a solução de três fatores para os dois 
casos. A variância explicada pelo conjunto de fatores foi semelhante nas duas amostras ( $45 \%$ e 44\%). A consistência interna dos fatores em ambas as amostras foi satisfatória (alfas acima de 0,77 , correlações inter-item superiores a 0,29 e correlações item-total maiores do que 0,45 ).

O item EB7 apresentou, na amostra de menor tempo de serviço, diferença inferior a 1,0 entre cargas fatoriais dirigidas a dimensões distintas. Tal resultado pode ser um indício de que esse item, por tratar de benefícios acumulados, é mais adequado nos casos de maior tempo de dedicação à organização. Contudo, com base nos resultados anteriores, não é considerada oportuna a exclusão do item EB7, que impactaria também na consistência interna do seu fator.

\section{Análise Fatorial Confirmatória}

Não obstante as análises exploratórias tenham indicado uma estrutura tridimensional para o entrincheiramento na organização, o debate presente na literatura sobre o fator LA, mencionado na introdução deste trabalho, justifica a comparação de dois modelos distintos por meio da análise fatorial confirmatória: o primeiro modelo considera o entrincheiramento organizacional como um construto trifatorial, incluindo a dimensão LA, que representa a percepção de limitação de alternativas originada externamente; e o segundo modelo parte da hipótese de que é um construto bidimensional, sendo a percepção de alternativas limitadasderivada das dimensões APS e ABI e originada por um processo intrínseco.

Para a avaliação dos modelos, três etapas foram seguidas: (a) teste e modificação dos modelos de mensuração em uma sub-amostra do total de participantes; (b) nova avaliação dos modelos re-especificados, a partir de uma segunda amostra independente da primeira (validação cruzada), a fim de testar a adequação dos ajustes executados; e (c) teste dos modelos estruturais com a variável latente de segunda ordem, utilizando a amostra completa, a fim de minimizar possíveis efeitos nos índices de ajuste causados pelos tamanhos das sub-amostras.

\section{Validações Cruzadas dos Modelos de Mensuração}

Essa etapa teve a finalidade de verificar se as modificações sugeridas pelos índices post hoc são generalizáveis. Foram utilizadas as mesmas sub-amostras empregadas na validação cruzada da análise exploratória.

O modelo tridimensional, especificado com os 22 itens restantes da etapa exploratória, foi superindentificado ( $\mathrm{GL}=202)$ e, após estimado, obteve índices de modificação post hoc que embasaram a adição de quatro covariâncias não previstas no modelo inicial. A primeira foi relativa aos erros dos itens ES7 e ES8, que têm em comum os relacionamentos formados na organização e que poderiam ser perdidos caso o indivíduo a deixasse. A segunda covariância acrescentada foi entre os erros dos itens EL3 e ES4, cujo foco é no conhecimento restrito a determinada realidade organizacional, que dificultaria a inserção do indivíduo em um novo emprego. $\mathrm{O}$ terceiro parâmetro foi adicionado entre os erros das variáveis EB3 e EB4, que colocam em evidência os riscos financeiros associados à saída da organização. Por fim, acrescentouse uma relação de covariância entre os erros dos itens EB1 e IL4. Interpreta-se que a estabilidade referida no item EB1 pode estar relacionada à dificuldade de ingresso em outra organização, a que se remete o item IL4. O fato de serem itens de fatores distintos não desaconselha a adição de um parâmetro, uma vez que as dimensões são correlacionadas e outros indicadores puros estão ligados às variáveis latentes (McDonald \& Ho, 2002).

No caso do modelo bidimensional, também super-identificado ( $\mathrm{GL}=89$ ), foram mantidos os 15 itens das dimensões APS e ABI, após a retirada do fator LA. Quando levantados os índices de modificação, observou-se que os dois parâmetros indicados para acréscimo foram os mesmos adicionados para os fatores APS e ABI no modelo tridimensional. Esse é mais um dado de que essas alterações têm suporte teórico e empírico. Na Tabela 2, são apresentados os índices encontrados para as duas estruturas, nas duas sub-amostras.

Nota-se que os índices melhoraram após as re-especificações e, em sua maioria, alcançaram os parâmetros esperados. Os modelos re-especificados, em ambas as estruturas (tri e bidimensional), apresentaram valores aproximados dos índices nas duas sub-amostras, sinalizando que as alterações geraram o mesmo padrão de aprimoramento nos dois grupos.

Embora possivelmente afetadas pelo tamanho da amostra e pela anormalidade dos dados, as diferenças de $\chi^{2}$ entre os modelos aninhados forneceram evidências adicionais de melhor ajuste após as modificações. Nas duas sub-amostras, as diferenças foram significativas, tanto no modelo tridimensional $\left[\chi^{2} \operatorname{dif}(4, n=359)=79,534\right.$ e $\chi^{2} \operatorname{dif}(4$, $n=361)=61,658]$ quanto no modelo bidimensional $\left[\chi^{2} \operatorname{dif}(2, n=359)=48,215\right.$ e $\left.\chi^{2} \operatorname{dif}(2, n=361)=42,727\right]$.

A comparação dos índices de parcimônia também confirma a maior adequação dos modelos re-especificados, sendo melhor o modelo que apresenta o menor valor. $\mathrm{Na}$ Tabela 2, são descritos entre parêntesis os valores da razão crítica entre cada índice (AIC e CAIC) e o número de parâmetros estimados, seguindo a recomendação de Pilati e Laros (2007).

Nas duas amostras, em ambas as estruturas, os parâmetros estimados apresentaram coeficientes altamente significativos. A única exceção ocorreu para o parâmetro acrescentado no modelo trifatorial entre os erros das variáveis EL3 e ES4 na segunda amostra, não significativo. As variáveis latentes apresentaram fortes correlações entre si, revelando a existência de uma variável de segunda ordem. Tendo em vista a significância estatística dos coeficientes e a manutenção, nas duas amostras, dos padrões de melhoria advindos das modificações, é possível afirmar que há validade cruzada dos modelos re-especificados. 
Rodrigues, A. C. A. \& Bastos, A. V. B. (2012). Entrincheiramento Organizacional: Construção e Validação da Escala.

Tabela 2

Índices de Ajuste do Modelo Tridimensional do Entrincheiramento para as Duas Amostras

\begin{tabular}{|c|c|c|c|c|c|c|c|c|}
\hline \multirow[t]{3}{*}{ Índice } & \multicolumn{4}{|c|}{ Amostra 1} & \multicolumn{4}{|c|}{ Amostra 2} \\
\hline & \multicolumn{2}{|c|}{ Modelo Inicial } & \multicolumn{2}{|c|}{ Re-especificado } & \multicolumn{2}{|c|}{ Modelo Inicial } & \multicolumn{2}{|c|}{ Re-especificado } \\
\hline & Tri & $\mathrm{Bi}$ & Tri & $\mathrm{Bi}$ & Tri & $\mathrm{Bi}$ & Tri & $\mathrm{Bi}$ \\
\hline NFI & 0,78 & 0,82 & 0,82 & 0,86 & 0,81 & 0,84 & 0,83 & 0,87 \\
\hline IFI & 0,86 & 0,88 & 0,90 & 0,92 & 0,88 & 0,89 & 0,91 & 0,92 \\
\hline CFI & 0,86 & 0,88 & 0,90 & 0,91 & 0,88 & 0,89 & 0,90 & 0,92 \\
\hline RMSEA & 0,06 & 0,07 & 0,05 & 0,06 & 0,06 & 0,07 & 0,05 & 0,06 \\
\hline GFI & 0,89 & 0,91 & 0,91 & 0,93 & 0,89 & 0,92 & 0,91 & 0,94 \\
\hline AGFI & 0,86 & 0,88 & 0,88 & 0,91 & 0,87 & 0,89 & 0,88 & 0,91 \\
\hline RMR & 0,13 & 0,14 & 0,11 & 0,12 & 0,13 & 0,13 & 0,12 & 0,12 \\
\hline PGFI & 0,72 & 0,68 & 0,72 & 0,68 & 0,73 & 0,68 & 0,72 & 0,68 \\
\hline AIC & $\begin{array}{l}582,8 \\
(12,4)\end{array}$ & $\begin{array}{l}309,1 \\
(10,0)\end{array}$ & $\begin{array}{l}511,3 \\
(10,0)\end{array}$ & $\begin{array}{c}264,9 \\
(8,0)\end{array}$ & $\begin{array}{l}571,0 \\
(12,2)\end{array}$ & $\begin{array}{c}300,2 \\
(9,7)\end{array}$ & $\begin{array}{l}517,4 \\
(10,1)\end{array}$ & $\begin{array}{c}261,49 \\
(7,9)\end{array}$ \\
\hline CAIC & $\begin{array}{l}812,3 \\
(17,3)\end{array}$ & $\begin{array}{l}460,5 \\
(14,9)\end{array}$ & $\begin{array}{l}760,3 \\
(14,9)\end{array}$ & $\begin{array}{l}426,0 \\
(12,9)\end{array}$ & $\begin{array}{l}800,8 \\
(17,0)\end{array}$ & $\begin{array}{l}451,8 \\
(14,6)\end{array}$ & $\begin{array}{l}766,7 \\
(15,0)\end{array}$ & $\begin{array}{r}422,82 \\
(12,8)\end{array}$ \\
\hline $\begin{array}{l}\chi^{2} \\
(\rho<0,001)\end{array}$ & $\begin{array}{l}488,810 \\
(g l=206)\end{array}$ & $\begin{array}{c}247,098 \\
(g l=89)\end{array}$ & $\begin{array}{l}409,276 \\
(g l=202)\end{array}$ & $\begin{array}{c}198,883 \\
(g l=87)\end{array}$ & $\begin{array}{l}477,029 \\
(g l=206)\end{array}$ & $\begin{array}{c}238,217 \\
(g l=89)\end{array}$ & $\begin{array}{l}415,371 \\
(g l=202)\end{array}$ & $\begin{array}{r}195,490 \\
(g l=87)\end{array}$ \\
\hline
\end{tabular}

Nota. Fonte: Dados da pesquisa.

Teste dos Modelos Estruturais

Todas as modificações estabelecidas nos modelos de mensuração foram mantidas nos testes dos modelos com a variável de segunda ordem, aplicados à amostra total. Os testes de adequação para ambos os modelos tri e bidimensional revelaram índices satisfatórios (Tabela 3).

Tabela 3

Índices de Ajuste dos Modelos Trifatorial e Bifatorial de Entrincheiramento Organizacional

\begin{tabular}{lcc}
\hline Índice & Modelo Trifatorial & Modelo Bifatorial \\
\hline NFI & 0,87 & 0,90 \\
IFI & 0,91 & 0,93 \\
CFI & 0,91 & 0,93 \\
RMSEA & 0,05 & 0,05 \\
GFI & 0,93 & 0,96 \\
AGFI & 0,92 & 0,94 \\
RMR & 0,096 & 0,096 \\
PGFI & 0,75 & 0,69 \\
AIC & $665,904(13,1)$ & $326,536(9,9)$ \\
CAIC & $950,516(18,6)$ & $510,697(15,5)$ \\
$\chi^{2}$ & 563,904 & 260,536 \\
$(p<0,001)$ & $(\mathrm{GL}=202)$ & $(\mathrm{GL}=87)$
\end{tabular}

Nota. Fonte: Dados da pesquisa.

No caso da estrutura tridimensional, somente o NFI não alcançou o valor mínimo necessário, mas os resultados do IFI, CFI e RMSEA sinalizam que o modelo possui um bom ajuste em comparação a outros modelos aninhados hipotéticos. Os valores do GFI e do AGFI indicam que o modelo é capaz de estimar uma matriz de covariância que explica significativamente a variância da matriz dos dados da amostra. Por fim, o RMR resultou em um valor abaixo de 1,0, que é considerado positivo, embora esse índice não possua um teste estatístico que estabeleça um ponto de corte. Sua interpretação foi complementada com a inspeção da matriz de resíduos padronizados, onde valores altos (acima de 2,0) foram encontrados. As variáveis EB1, EL3 e EB3 foram identificadas como as mais críticas, uma vez que apresentam resíduos com diferentes variáveis. Nota-se que as três já foram alvo de re-especificação, indício de que podem ser itens redundantes ou com problemas na elaboração do enunciado.

Todos os parâmetros estimados no modelo são significativos, conforme observado na Figura 1. A dimensão APS é a mais representativa do modelo, com $83 \%$ de sua variância explicada pelo entrincheiramento organizacional. As três variáveis latentes de primeira ordem mostraramse boas preditoras dos itens, uma vez que as cargas fatoriais estiveram entre 0,41 e 0,69 , e as variâncias associadas aos erros dos itens estiveram entre 0,16 e 0,47 . As covariâncias adicionadas na re-especificação do modelo de mensuração apresentaram significância estatística e valores moderados. Com base nos índices de ajuste e nos coeficientes estimados, o modelo tridimensional do entrincheiramento é considerado aceitável. 


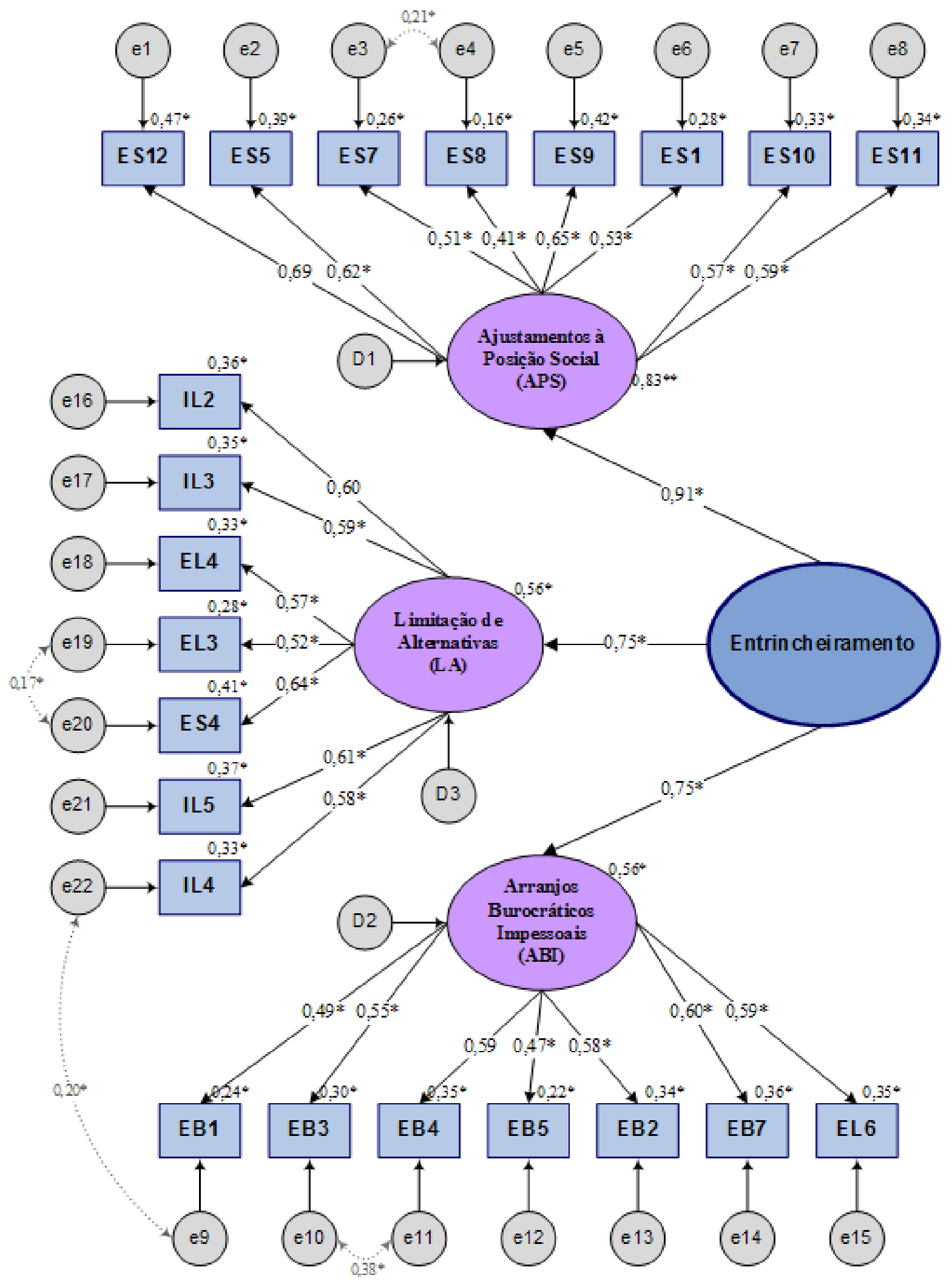

Figura 1. Modelo re-especificado do entrincheiramento organizacional (3 fatores).

Nota. Fonte: Dados da pesquisa. ${ }^{*} p<0,001 ;{ }^{*} p<0,01$.

Com relação ao modelo bidimensional, observou-se igualmente que todos os índices de ajuste e coeficientes estimados foram satisfatórios e significativos, conforme apresentado na Tabela 3 e na Figura 2. 


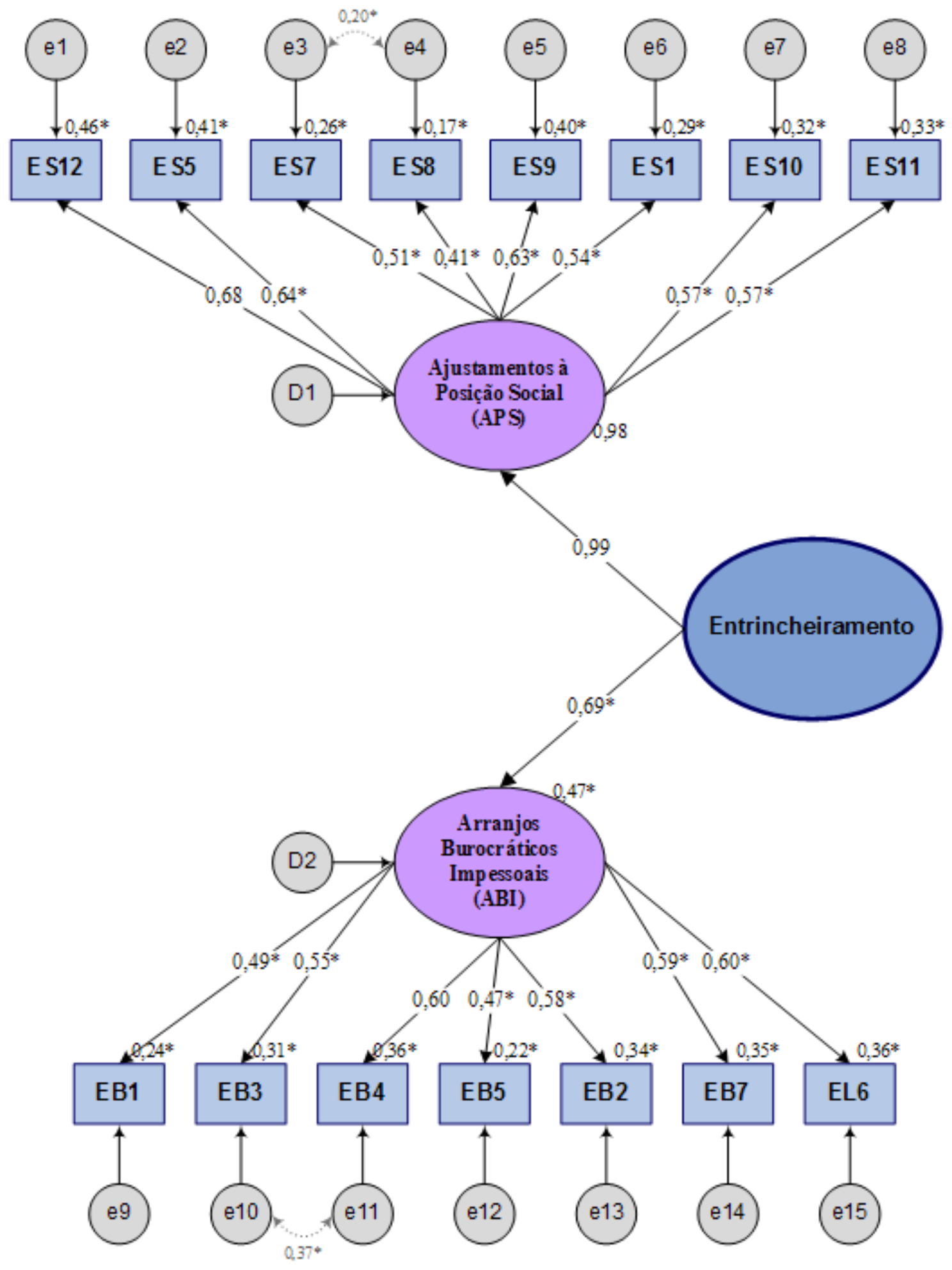

Figura 2. Modelo re-especificado do entrincheiramento organizacional (2 fatores). Nota. Fonte: Dados da pesquisa. ${ }^{*} p<0,001$.

O exame da matriz de resíduos padronizados condiz com o baixo valor do RMR encontrado e reforça a interpretação de bom ajuste do modelo. Mais uma vez, o item EB1 obteve a maior frequência de resíduos. Uma análise de sua formulação aponta para uma baixa precisão da assertiva, pois a ameaça à estabilidade pode ser interpretada de diferentes formas. Portanto, esse item merece ser revisado ou excluído da escala de entrincheiramento organizacional em estudos futuros. 


\section{Discussão}

Comparação dos Modelos Concorrentes:

Trifatorial x Bifatorial

A comparação dos dois modelos apresentados para a estrutura do entrincheiramento organizacional considerou os indicadores de ajuste, já que ambos apresentam coeficientes estimados semelhantes e significativos. Observa-se que os valores são aproximados, e são satisfatórios os índices de ajuste comparativo, proporção de variância explicada e ajuste baseado em resíduos. Notase que o modelo bifatorial apresenta os menores valores para o AIC e para o CAIC, contudo o PGFI é menor do que o obtido pelo modelo trifatorial, justificado pelo menor número de graus de liberdade da solução bifatorial (Ullman, 2007). Ainda assim, interpreta-se que os dois modelos apresentam indicadores empíricos muito semelhantes, e que os valores dos índices de parcimônia não são suficientes para a indicação do modelo mais apropriado.

O emprego da técnica de modelagem de equações estruturais é oportuno para confrontar modelos e investigar qual das hipóteses é capaz de estimar uma matriz de correlação da população que seja mais consistente com a matriz dos dados coletados (Ullman, 2007). Contudo, em casos de índices igualmente positivos para os dois modelos, recorre-se a um debate teórico para a determinação de qual seja o melhor (Quintana \& Maxwell, 1999).

O ponto de partida para a discussão sobre o melhor modelo é a pertinência do fator LA como um componente do construto entrincheiramento organizacional. É importante lembrar que esse argumento tem origem nos estudos sobre o comprometimento e nas duas subdimensões identificadas para a base de continuação sacrifícios pessoais e limitação de alternativas. Alguns autores defendem que a falta de alternativas percebida pelo trabalhador seja considerada um antecedente, visto que impacta na relação do indivíduo com a organização, favorecendo sua decisão por permanecer (K. D. Carson \& P. P. Carson, 2002; Ko et al., 1997; Powell \& Meyer, 2004). A permanência, por sua vez, é entendida como um possível consequente do comprometimento, assim como empenho extra, desempenho, cidadania organizacional, entre outros. Não se justifica que, dentre tantas variáveis critério, a noção de permanência seja enfatizada a ponto de ter um determinante direto - limitação de alternativas - como constituinte do conceito de comprometimento.

Ao tratar do entrincheiramento organizacional, em contrapartida, vê-se que os argumentos para que LA não seja parte do comprometimento servem como respaldo para sua integração ao construto de entrincheiramento. Embora a noção de permanência não deva ser considerada uma dimensão constitutiva do primeiro, está fortemente ligada ao segundo, não a permanência por vontade do trabalhador, mas a que é necessária, devido às condições que o cercam: as vantagens recebidas, o tempo e esforço despendidos, o processo adaptativo, perdas que teria caso saísse, e que são potencializadas por não perceber outras alternativas disponíveis. Assim, a percepção de falta de alternativas não é entendida apenas como um antecedente para a permanência, mas como uma condição no processo de entrincheiramento do trabalhador. Por esse motivo, considera-se como mais adequado o modelo tridimensional do entrincheiramento organizacional.

\section{Considerações Finais}

O objetivo deste trabalho foi construir e validar uma escala de entrincheiramento organizacional. Os resultados encontrados indicam boas propriedades psicométricas e adequação estatística e conceitual da estrutura de três fatores, formada pelas dimensões APS (ajustamentos à posição social), ABI (arranjos burocráticos impessoais) e LA (limitação de alternativas). Foram verificadas validade cruzada e generalizabilidade deste modelo após aplicação de técnicas específicas com a utilização de análises fatoriais exploratória e confirmatória. Os resultados corroboram, ainda, o quadro teórico desenvolvido por Rodrigues e Bastos (2011) para o conceito de entrincheiramento com foco na organização.

Algumas limitações podem ser salientadas para um tratamento cauteloso dos resultados aqui obtidos e para o aprimoramento de investigações futuras. A amostra utilizada para as análises é composta em sua maioria por trabalhadores jovens, com pouco tempo de empresa e alta escolaridade, fato que reduz a variabilidade da amostra. Além disso, as violações à normalidade dos dados, embora comuns nos estudos em ciências sociais e influenciados por desejabilidade social, podem ter impactado nos resultados obtidos para os índices de ajuste e para as matrizes de resíduos dos modelos testados (Pilati \& Abbad, 2005).

O emprego de modelagem de equações estruturais, que é considerado uma força do estudo devido à sua sofisticação metodológica, fornece limitações ligadas à falta de consenso em relação a alguns procedimentos e decisões necessárias e à inexistência de um padrão estabelecido para o relato dos resultados (Weston \& Gore, 2006). Diante da ameaça à comparação dos resultados, a matriz de correlação entre as variáveis testadas, médias e desvios padrões podem ser disponibilizadas mediante contato com os autores, a fim de viabilizar re-testagens por pesquisadores interessados.

Por fim, algumas sugestões para estudos futuros podem ser enunciadas. Diante da necessidade de um maior refinamento conceitual e empírico para o construto de entrincheiramento organizacional, recomenda-se que a avaliação psicométrica da escala aqui elaborada seja repetida em outras amostras, para que novos insumos de modificação e melhoria dos itens sejam obtidos. Outra fonte de informações para o aprimoramento do conteúdo da escala consiste na aplicação de estudos qualitativos que investiguem o conceito de entrincheiramento organizacional para os trabalhadores, possibilitando, ainda, 
verificar se os atores organizacionais incluem na definição do construto a noção de alternativas limitadas. A análise dessa dimensão poderá ser favorecida também por estudos descritivos que avaliem o nível de entrincheiramento organizacional em amostras de diferentes segmentos ou ocupações com maior ou menor oferta de trabalho no mercado. Ainda, para o desenvolvimento do construto, recomenda-se que pesquisas sejam conduzidas para avaliar seus antecedentes e consequentes e que sejam aplicados, também, delineamentos longitudinais, que permitam uma maior compreensão do processo de estabelecimento desse tipo de vínculo. Até que sejam reunidas informações teóricas e empíricas suficientes para uma decisão mais precisa acerca da dimensionalidade do entrincheiramento organizacional, recomenda-se fortemente que estudos quantitativos utilizem a escala com os três fatores (APS, ABI e LA).

Este trabalho cumpre o papel de iniciar uma agenda de pesquisa que fornecerá novos rumos para os estudos sobre vínculos entre o indivíduo e a organização: o desenvolvimento teórico e empírico do construto de entrincheiramento organizacional e o avanço das investigações que poderão apoiar uma maior delimitação do conceito de comprometimento organizacional. Com uma maior precisão dos conceitos e instrumentos de medida, espera-se assegurar resultados mais válidos sobre os vínculos do trabalhador, que possam gerar novos estudos na área e que sejam base para práticas de desenvolvimento e gestão dos vínculos nas organizações.

\section{Referências}

Bastos, A. V. B., Siqueira, M. M. M., Medeiros, C. A., \& Menezes, I. G. (2008). Comprometimento organizacional. In M. M. M. Siqueira (Ed.), Medidas de comportamento organizacional: Ferramentas de diagnóstico e de gestão (pp. 49-95). Porto Alegre, RS: Artmed.

Becker, H. S. (1960). Notes on the concept of commitment. The American journal of Sociology, 66, 32-40.

Blau, G. (2001). Testing the discriminant validity of occupational entrenchment. Journal of Occupational and Organizational Psychology, 74, 85-93.

Blau, G., \& Holladay, E. B. (2006). Testing the discriminant validity of a four-dimensional occupational commitment measure. Journal of Occupational and Organizational Psychology, 79, 691-704.

Carson, K. D., \& Carson, P. P. (2002). Differential relationships associated with two distinct dimensions of continuance commitment. International Journal of Organization Theory and Behavior, 5(3/4), 359-381.

Carson, K. D., Carson, P. P., \& Bedeian, A. G. (1995). Development and construct of a career entrenchment measure. Journal of Occupational and Organizational Psychology, 68, 301-320.

Clark, L. A., \& Watson, D. (1995). Constructing validity: Basic issues in objective scale development. Psychological Assessment, 7(3), 309-319.

Cooper-Hakim, A., \& Viswesvaran, C. (2005). The construct of work commitment: Testing an integrative framework. Psychological Bulletin, 131(2), 241-259.
Gondim, S. M. G., Borges-Andrade, J. E., \& Bastos, A. V. B. (2010). Psicologia do Trabalho e das Organizações: Produção científica e desafios metodológicos. Psicologia em Pesquisa, 4(2), 84-99.

Hair, J. F., Jr., Anderson, R. E., Tatham, R. L., \& Black, W. C. (2005). Análise multivariada de dados. Porto Alegre, RS: Bookman.

Klein, H. J., Molloy, J. C., \& Cooper, J. C. (2009). Conceptual foundations: Construct definitions and theoretical representations of workplace commitments. In H. J. Klein, T. Becker, \& J. P. Meyer (Eds.), Commitment in organizations: Accumulated wisdom and new directions (pp. 3-36). New York: Routledge Academic.

Ko, J. W., Price, J. L., \& Mueller, C. W. (1997). Assessment of Meyer and Allen's three-component model of organizational commitment in South Korea. Journal of Applied Psychology, 82, 961-973.

Laros, J. A. (2005). O uso da análise fatorial: Algumas diretrizes para pesquisadores. In L. Pasquali (Ed.), Análise fatorial para pesquisadores (pp. 163-184). Brasília, DF: Laboratório de Pesquisa em Avaliação e Medida.

Magalhães, M. O. (2008). Generatividade e vínculos com a carreira e a organização: Problematizando as bases do comprometimento organizacional. Trabalho apresentado no III Congresso Brasileiro de Psicologia Organizacional e do Trabalho, Florianópolis, SC.

McDonald, R. P., \& Ho, M. R. (2002). Principles and practice in reporting structural equation analyses. Psychological Methods, 7(1), 64-82.

McGee, G., \& Ford, R. (1987). Two (or more?) dimensions of organizational commitment: Reexamination of the affective and continuance commitment scales. Journal of Applied Psychology, 72, 638-642.

Menezes, I. G. (2006). Escalas de intenções comportamentais de comprometimento organizacional (EICCO): Concepção, desenvolvimento, validação e padronização. (Dissertação de Mestrado não-publicada). Universidade Federal da Bahia, Salvador, BA.

Meyer, J. P., Allen, N. J., \& Smith, C. A. (1993). Commitment to organizations and occupations: Extension and test of a three-component conceptualization. Journal of Applied Psychology, 78(4), 538-551.

Mowday, R. T., Porter, L. W., \& Steers, R. M. (1982). Employeeorganization linkages: The psychology of commitment, absenteeism, and turnover. New York: Academic Press.

Osigweh, C. A. B. (1989). Concept fallibility in organizational science. The Academy of Management Review, 14(4), 579594.

Pasquali, L. (1999). Instrumentos psicológicos: Manual prático de elaboração. Brasília, DF: Laboratório de Pesquisa em Avaliação e Medida.

Pilati, R., \& Abbad, G. (2005). Análise fatorial confirmatória da escala de impacto do treinamento no trabalho. Psicologia: Teoria e Pesquisa, 21(1), 43-51.

Pilati, R., \& Laros, J. A. (2007). Modelos de equações estruturais em Psicologia: Conceitos e aplicações. Psicologia: Teoria e Pesquisa, 23(2), 205-216.

Powell, D. M., \& Meyer, J. P. (2004). Side-bet theory and the three-component model of organizational commitment. Journal of Vocational Behavior, 65, 157-177.

Quintana, S. M., \& Maxwell, S. E. (1999). Implications of recent developments in structural equation modeling for counseling psychology. The Counseling Psychologist, 27(4), 485-527. 
Rego, A. (2003). Comprometimento organizacional e ausência psicológica: Afinal, quantas dimensões? Revista de Administração de Empresas, 45(4) 25-35.

Rodrigues, A. C. A. (2009). Do comprometimento de continuação ao entrincheiramento organizacional: O percurso de validação da escala e análise da sobreposição entre os construtos. (Dissertação de Mestrado não-publicada). Universidade Federal da Bahia, Salvador, BA.

Rodrigues, A. C. A., \& Bastos, A. V. B. (2010). Problemas conceituais e empíricos na pesquisa sobre comprometimento organizacional: Uma análise crítica do modelo tridimensional de J. Meyer e N. Allen. Revista de Psicologia Organizacional e do Trabalho, 10(2), 129-144.

Rodrigues, A. C. A., \& Bastos, A. V. B. (2011). Entrincheiramento organizacional: Proposta de um novo vínculo indivíduo-organização. In J. C. Zanelli, N. Silva, \& S. R. Tolfo (Eds.), Processos psicossociais nas organizações e no trabalho (pp. 161-178). São Paulo, SP: Casa do Psicólogo.

Scheible, A. C. F., \& Bastos, A. V. B. (2006). Comprometimento com a carreira: Explorando o conceito de entrincheiramento. Trabalho apresentado no XXX Encontro da Associação Nacional de Pós-Graduação e Pesquisa em Administração, Salvador, BA.

Scheible, A. C. F., Bastos, A. V. B., \& Rodrigues, A. C. A. (2007). Comprometimento e entrincheiramento: Integrar ou reconstruir? Uma exploração das relações entre estes construtos à luz do desempenho. Trabalho apresentado no XXXI Encontro da Associação Nacional de Pós-Graduação e Pesquisa em Administração, Rio de Janeiro, RJ.

Solinger, O. N., Olffen, W., \& Roe, R. A. (2008). Beyond the three-component model of organizational commitment. Journal of Applied Psychology, 93(1), 70-83.

Ullman, J. B. (2007). Structural equation modeling. In B. G. Tabachnick \& L. S. Fidell, Using multivariate statistics $\left(5^{\text {th }}\right.$ ed, pp. 676-780). New York: Pearson Education.

Weston, R., \& Gore, P. A., Jr. (2006). A brief guide to structural equation modeling. The Counseling Psychologist, 34(5), 719-751.

Zwick, W. R., \&Velicer, W. F. (1986). Comparision of five rules for determining the number of components to retain. Psicological Bulletin, 99(3), 432-442. 